\title{
Métodos e ferramentas para o processo de planejamento docente no contexto dos estudos da área de Design da Aprendizagem
}

Gabrielle dos Santos, Universidade Feevale, gabrielle@feevale.br Patrícia Scherer Bassani, Universidade Feevale, patriciab@feevale.br

Resumo: O planejamento docente se caracteriza a partir de três etapas complementares: a definição dos resultados pretendidos, a proposição das atividades de ensino e as atividades avaliativas. Estudos na área de Design da Aprendizagem focam na proposição de ferramentas e métodos para guiar o planejamento e o compartilhamento de atividades de aprendizagem. Assim, este estudo tem por objetivo identificar quais as ferramentas e os métodos usados no contexto do planejamento docente a partir dos estudos na área de Design da Aprendizagem. A metodologia de pesquisa envolveu uma revisão sistemática de literatura, a partir de três bases de dados. Como resultados foram identificadas 12 ferramentas e métodos produzidos entre os anos de 2007 e 2018, que podem ser utilizados no processo de planejamento docente.

Palavras-chave: design da aprendizagem; planejamento docente; ensino superior; experiências de aprendizagem; alinhamento construtivo

\section{Tools and methods used on the process of teaching planning based on the context of studies in the Learning Design area}

Abstract: The teaching planning process is characterized by three complementary steps: the definition of desired learning outcomes, the proposal of teaching activities and the assessment. Studies in the area of Learning Design focus on proposing tools and methods to guide the planning and sharing of learning activities. Thus, this study aims to identify which tools and methods are used in the context of teacher planning based on Learning Design studies. The methodology of the research involved a systematic literature review, based on three databases. Results revealed a set of 12 tools and methods produced between 2007 and 2018 which can be used in the teaching planning process.

Keywords: learning design; teaching planning; higher education; learning experiences; constructive alignment

\section{Introdução}

O planejamento docente, apoiado na teoria do Alinhamento Construtivo de Biggs (1996), tem o aluno como centro do processo de aprendizagem, sendo este o ponto chave e de partida para um planejamento mais assertivo. Esta teoria sugere o alinhamento harmônico entre todos os itens elementares que devem ser considerados em um planejamento no contexto educativo, a saber: resultados pretendidos da aprendizagem (intended learning outcomes - ILO), atividades de ensino e aprendizagem (teaching learning activities - TLA) e as atividades de avaliação (assessment task-AT).

Nesta perspectiva, o docente deve ter em mente o que o aluno deve aprender e quais competências ele estará apto a executar após o seu processo formativo. Este pensamento deve ocorrer anteriormente à elaboração dos conteúdos, bem como das atividades de ensino, pois, conforme Biggs (1996), as duas últimas etapas do planejamento (atividades e avaliação) só serão assertivas quando estiverem em total harmonia com a primeira etapa. Assim, todas as experiências de aprendizagem elaboradas devem estar alinhadas com as competências/resultados previstos. A taxonomia de Bloom 
et al. (1956), que disponibiliza diversos graus de complexidade por meio de objetivos cognitivos (expressados em verbos) a serem explorados pelo docente, pode ser utilizada como guia na primeira etapa do processo de planejamento, que envolve a definição dos resultados de aprendizagem.

As três etapas do alinhamento construtivo, propostas por Biggs (1996), dão suporte ao docente no momento do planejamento, indicam o caminho a ser traçado de forma alinhada e relevante para fomentar a aprendizagem dos alunos. Além disso, indica o caminho a ser seguido pelo docente ao conduzir a práxis.

Como prática decisória e imprescindível, o planejamento docente deverá ser feito e registrado diariamente, podendo ser compartilhado e acessível a todos os públicos. Assim, deverá ser elaborado de forma clara e objetiva, a fim de promover novas experiências de aprendizagem (MENEGOLLA; SANT'ANNA, 2014).

Pesquisas na área da Educação indicam a necessidade de se promover, em sala de aula, atividades voltadas à prática discente, que proporcionam o protagonismo dos alunos, por meio de metodologias inovadoras (MORAN, 2015). Estas devem instigá-los a se colocarem como agentes principais de seu processo formativo. Porém, para que este processo se desenvolva, cabe uma reflexão quanto a práxis docente. Desta forma, é preciso compreender o processo de planejamento, desde sua concepção, com a definição de competências, objetivos, atividades, recursos e avaliação.

Na perspectiva do Alinhamento Construtivo (BIGGS, 1996), o processo de aprendizagem, voltado ao aluno, nos faz repensar e entender como o ensino se comporta hoje nos espaços de aprendizagem, que acontecem em qualquer tempo e em qualquer lugar. A aprendizagem é algo orgânico e constante ao ser humano, estamos destinados a aprender sempre, sem mesmo estarmos pré-dispostos para tal. Mesmo isto posto, a educação ainda é um campo muito restrito e tradicional em seus processos e práticas.

O Design da Aprendizagem (ou Learning Design) é uma área de pesquisa que foca no desenvolvimento de ferramentas, métodos e abordagens que buscam auxiliar o professor no planejamento, na utilização e no compartilhamento de atividades de aprendizagem que façam uso efetivo das diferentes tecnologias digitais.

O Design da Aprendizagem (DA) se organiza a partir de três pilares: orientação, representação e compartilhamento. Sob a perspectiva da orientação, tem por objetivo auxiliar os docentes na elaboração e na proposição de atividades de aprendizagem com tecnologias, envolvendo formação em tecnologias digitais e orientação no planejamento como um todo. Sob a perspectiva da representação, foca no desenvolvimento e na proposição de formas para representar/documentar atividades de aprendizagem. Por fim, sob a perspectiva do compartilhamento, visa disponibilizar as representações para que outros docentes possam ter acesso a diferentes ideias de práticas de ensino (DALZIEL et al., 2016).

Este estudo busca sistematizar pesquisas já desenvolvidas sob a perspectiva da orientação, a fim de identificar ferramentas e métodos que estão sendo utilizadas para orientar o processo de planejamento docente, com base nos estudos de DA.

O artigo está assim organizado: na seção 2 apresentamos o delineamento metodológico do estudo; na seção 3, os resuldados, e na seção 4, a discussão. Por fim, as considerações finais, seguidas das refêrências deste estudo.

\section{Metodologia}

Este estudo se articula ao projeto de pesquisa "Práticas educativas em/na rede: autoria e colaboração no desenvolvimento e atividades de aprendizagem com tecnologias 
digitais 1 ", que tem por objetivo propor um ambiente para o registro e o compartilhamento de atividades de aprendizagem com tecnologias digitais.

A pesquisa foi inspirada na metodologia de revisão sistemática de literatura proposta por Kitchenham e Charters (2007). O processo de pesquisa iniciou com a definição da questão de pesquisa (RQ - research question): Quais ferramentas e métodos estão sendo utilizados para orientar o processo de planejamento docente?

A coleta de dados foi realizada em três bases de dados, Scopus, Unique ${ }^{2}$ e Computers \& Education", a partir das seguintes palavras-chave: "learning design" e "design da aprendizagem".

A pesquisa retornou um conjunto de 1.413 resultados, envolvendo artigos, dissertações e teses, assim organizados: 677 da base Unique; 636 da Scopus; 100 da Computers \& Education.

O conjunto de textos foi exportado para o ambiente on-line Rayyan QCRI ${ }^{4}$ (https://rayyan.qcri.org/). Novos termos foram aplicados para refinar a busca: framework/estrutura (691), tools/ferramenta (200), higher education/ensino superior (193), teacher/professor (162), learning experiences/experiências de aprendizagem (71) e scenarios/cenários (29). Os termos estabelecidos estão ligados diretamente ao planejamento docente, no que diz respeito a sua elaboração, construção, e ou reconstrução, por meio do design da aprendizagem, conforme objeto deste artigo.

Esta segunda pesquisa reduziu o conjunto de textos para um total de 1.346. A redução não foi significativa em relação ao conjunto inicial. Portanto, foi necessário realizar uma verificação manual deste novo conjunto. Dessa forma, essa nova etapa envolveu a leitura de resumos e de palavras-chave.

Um dos três pilares do DA é a orientação (guidance), foco de interesse deste estudo. Dessa forma, os critérios de inclusão para a seleção dos artigos foram elencados a partir dessa perspectiva. A orientação (OR), segundo Larnaca (2013), visa dar suporte ao educador para criar/elaborar (ou até mesmo remixar) as práticas pedagógicas, refletir sobre o processo ensino-aprendizagem, o contexto em que o aluno está inserido, projetando assim, atividades inovadoras e eficazes, que buscam dar protagonismo ao discente e torná-lo agente de seu processo de aprendizagem. Cabe destacar que, por vezes, a orientação pode estar incorporada à representação, conforme citado no documento Larnaca Declaration (2013).

Por fim, foram selecionados um total de 13 artigos com foco em ferramentas e/ou métodos utilizados para orientar o processo de planejamento docente. Esses artigos foram lidos na íntegra ( 1 artigo escrito em português, 1 em espanhol e o restante em inglês).

\section{Resultados}

Dentre os treze artigos analisados foram identificados ferramentas e métodos diversificados para orientar a elaboração de novas práticas, por meio do planejamento docente.

\subsection{Métodos}

O método desenvolvido por Shaver (2017), em formato de reunião, se desdobra em três etapas, com a finalidade de planejar aulas on-line. O LD meeting busca auxiliar

\footnotetext{
${ }^{1} \mathrm{O}$ projeto está aprovado no Comitê de Ética protocolo número: CAAE: 05576818.8.0000.5348

${ }^{2}$ Sistema unificado da Universidade Feevale, que integra diversas bases de dados on-line.

${ }^{3}$ Esse periódico foi selecionado pelo seu foco interdisciplinar na área de tecnologia educacional. Além disso, o periódico é classificado como A nas áreas de Educação (A2), Ciência da Computação (A1) e Interdisciplinar (A2) no Qualis da Capes, e seu fator de impacto é 2018:5.627.

${ }^{4}$ Rayyan é desenvolvido pelo Qatar Computing Research Institute (Data Analytics)

V. $18 \mathrm{~N}^{\circ} 1$, julho, 2020

DOI: 
os docentes na transferência de experiências presenciais para as práticas on-line. $\mathrm{O}$ modelo busca, por meio de storyboard, auxiliar os alunos a organizarem o trabalho do curso. Também incentiva a construção de atividades baseadas em perguntas, atividades de expressão de linguagem, fóruns e blogs, priorizando sempre a interação e a colaboração entre os alunos. A autora destaca, ainda, a resistência por parte dos docentes, e a frustração na utilização de tecnologias digitais em seus planejamentos. Entretanto, resultados apontam para diversos benefícios como: explicação delineada dos procedimentos, redução da frustração em desenvolver cursos on-line e aumento da produtividade na conclusão dos cursos.

Georgsen (2011), elaborou o método Design Colaborativo de e-learning (CoED) que foi utilizado em workshops para a formação continuada de docentes na Dinamarca. O método tem como objetivo formalizar o design e externalizar as soluções e ideias para recursos e atividades de ensino. Também visa a construção colaborativa, propiciando uma dimensão global dos projetos de forma pedagógica e podendo ser compartilhada com os pares. Como pontos negativos, a autora identificou que, mesmo propiciando um momento de planejamento coletivo, alguns professores optaram por trabalharem sozinhos ou elencaram um projeto de determinado professor para ser trabalhado pelo grupo, não gerando novas propostas interdisciplinares.

Buus e Georgsen (2018), desenvolveram um método inspirado nos trabalhos anteriores de Georgsen e Nyavang (2007) e Georgsen (2011), com algumas variações e evoluções. O CoED é um conjunto de diretrizes que orienta por meio de workshops a criação ou recriação de projetos de aprendizagem digital, tanto em cursos completos como em módulos de aprendizagem, ou em atividades educacionais. A proposta envolve também diversas granularidades (atividade, projeto, curso). O ponto forte destacado pelos autores, ao utilizar a metodologia embasada no DA, é a negociação e a colaboração da visão pedagógica que é feita entre os pares, as discussões são enriquecedoras para a elaboração das práticas, tornando-as mais flexíveis e melhor estruturadas. Ao final das testagens, foram destacados diversos resultados, como: alguns docentes frequentaram mais os workshops do que necessário e estipulado pela instituição de ensino superior, também receberam devolutiva do quão relevante foram as discussões com os pares no momento de planejar novas práticas. Os autores destacaram, como ponto negativo, que alguns docentes evidenciaram que, devido à vasta experiência docente, não é necessário repensar sua filosofia de ensinar. Também como pontos negativos foram destacados: a falta de experiência na área da tecnologia da informação por parte dos docentes, falta de imaginação tecnológica e a falta de confiança em experimentar novos métodos de ensino.

Weitze (2017), criou um método pedagógico, denominado IT - Pedagogical Think Tank, com o intuito de promover a colaboração e desenvolvimento de novos projetos de aprendizagem, mediados por vídeo-aulas síncronas, de forma híbrida, que possibilita aos estudantes adultos escolherem assistir de casa a aula ou irem até a IES. O método foi validado com muitos professores, que indicaram ser ótimo para repensar o fazer docente. Ela também promoveu ações para os professores pensarem pedagogicamente sobre as atividades propostas. Também proporcionou, segundo o autora, um engajamento em outros professores que não estavam participando da pesquisa.

Capuano et. al. (2014), propõem um modelo adaptativo ao já existente método Intelligent Web Teacher (IWT). O modelo visa descrever contextos de aprendizagem, baseado na ontologia que, por sua vez, permite a representação de um domínio de ensino. Segundo os autores, a plataforma se mostrou bem estruturada no que diz respeito a recomendação de conteúdos, e em técnicas adaptativas. Também indica, ser um dos poucos no mercado que lida com contextos de aprendizagem. 


\subsection{Ferramentas}

Assis e Almeida (2017), no que diz respeito ao planejamento docente, indicam a dificuldade dos docentes em incluir tecnologias digitais em suas práticas e até mesmo metodologias inovadoras, que estão em evidência no cenário educativo. A inclusão de tecnologias, digitais ou analógicas, ainda é restrito na educação básica e até mesmo no ensino superior. Ainda há paradigmas a serem quebrados na educação com o uso de tecnologias, pois é necessário incorporá-las ao processo de ensinoaprendizagem. Para tal, as autoras analisaram a ferramenta Learning Design Support Environment (LDSE). Essa ferramenta, que foca no planejamento no contexto do ensino superior, tem como objetivo auxiliar no desenvolvimento de atividades de ensino, desde sua concepção, recursos entre outros, até as avaliações. As atividades propostas são feitas de forma colaborativa com os docentes, com vistas ao compartilhamento on-line. Também visa ser um ambiente de apoio aos professores para compartilhar, comparar e discutir com os pares, articulando princípios de aprendizagem aos processos de planejamento. As atividades de ensino podem ser elaboradas por módulo, sessão e/ou atividades, com níveis diferentes em cada processo. A partir dos estudos realizados no uso do ambiente, as autoras verificaram que os docentes enxergaram potencial na ferramenta de DA, uma vez que apresenta de forma visual os atributos necessários ao planejamento de ensino. Identificou-se a pré-disposição dos docentes para o uso das ferramentas on-line de DA para elaboração e trocas de experiências.

Dobozy (2017), também destaca a urgência do ensino superior em superar suas práticas tradicionais e transcender para um ensino adequado aos alunos do século XXI, diante das novas competências que são esperadas. A autora propõe uma ferramenta de elaboração de atividades de ensino, denominado Bono LAMS, que visa a transdisciplinaridade, desde a concepção da atividade, produção de objetos de aprendizagem, até a avaliação. Dobozy (2017) constatou que foi difícil engajar os docentes na proposta, mas os que participaram ativamente obtiveram resultados excelentes nos feedbacks dos alunos, reconheceram as práticas interativas como produtivas para o processo de aprendizagem. Quanto aos docentes participantes, indicaram facilidade em promover novas atividades e utilizar a tecnologia em suas práticas.

A ferramenta Higher Education Learning Design Framework (HELD) elaborada por Holdsworth e Hegarty (2016), vem com a proposta de identificar como se ministra aulas presenciais, entendendo que a educação centrada no professor está defasada. Compreendem que o aluno também é ator ativo em seu processo de aprendizagem, sendo protagonista de sua jornada. Argumentam que os docentes precisam envolvê-los em suas práxis, ouvindo-os e assim, repensando a prática docente, para haver uma aprendizagem significativa e prazerosa, a partir das vivências e bagagens pessoais dos discentes. Também trazem a importância de serem elaborados objetivos pensados ao público alvo, bem como as temáticas e conteúdos que serão abordados. A ferramenta é norteada por questões que instigam o projeto de aprendizagem.

Katsamani e Retalis (2013) desenvolveram a ferramenta denominada CADMOS, que visa apoiar o trabalho dos profissionais da educação, tornando-os designers. Após testarem diversas ferramentas elencaram 5 requisitos importantes a serem considerados pelo Design da Aprendizagem, que são: usabilidade, orientação, formalização, neutralidade pedagógica e flexibilidade do design. Estes serviram de base para a elaboração do CADMOS, que dispõe de duas camadas: (1) criação de atividades de aprendizagem conceitual e (2) criação de fluxo/orquestração das atividades. A ferramenta é voltada para iniciantes com pouca expertise na área de DA. A ferramenta está em 
constante atualização, conforme citam os autores. Entre as atualizações futuras está sendo pensada a possibilidade de exportarem os DA em formatos apropriados para o Moodle.

Pozzi e Persico (2013), propõem, por meio do conceito Computer-Supported Collaborative Learning, a ferramenta denominada 4Ts, para auxiliar professores no processo de planejamento pedagógico. A ferramenta foi baseada no conceito pedagogical planners da autora Conole (2013). A partir de estudos feitos na área, os autores identificaram que as ferramentas disponíveis não fornecem as orientações necessárias para tomar decisões referentes às atividades. Assim, propuseram a ferramenta 4Ts com quatro dimensões, que visa projetar e estruturar um aprendizado on-line e colaborativo. As quatro dimensões são: tarefa, equipe(s), tempo e tecnologia. Após algumas testagens, identificaram que a ferramenta ajudou a visualização de todas as dimensões, bem como auxiliou os docentes no desenho das atividades e tecnologias.

Churchill, King e Fox (2013), desenvolveram o modelo Resources-ActivitySupport-Evaluation (RASE) que tem como objetivo auxiliar os professores na elaboração de módulos de aprendizagem. Tem como eixo principal a elaboração de atividades avaliativas que incentivem a criação de recursos e artefatos tecnológicos, como forma de verificar a aprendizagem. A ferramenta também visa promover o protagonismo do aluno, com atividades que tenham a tecnologia como apoio à práxis docente, e que estas estejam centradas no aluno e não somente no professor, como na educação tradicional. O modelo é composto por 4 componentes: (1) recursos (2) atividade (3) suporte (4) avaliação.

Gros, Escofet e Martí (2016) propuseram uma ferramenta embasada no design da aprendizagem um pouco diferenciada das demais já apresentadas. Trata-se de um modelo que tem como ponto chave a orientação para a tomada de decisões no planejamento educacional, a partir de problemas reais que resultam em padrões pedagógicos. Denominada de Design2Learn, a proposição do modelo reuniu professores, pesquisadores e alunos para compreenderem quais problemas educacionais estavam em evidência, assim buscaram, por meio da ferramenta, soluções para estes problemas, por meio de práticas inovadoras e de fácil aplicação para as demais disciplinas, sendo acessível e possível para qualquer cenário. O objetivo central é proporcionar aos professores a visualização das abordagens e estratégias de ensino aplicadas a qualquer componente curricular e que podem ser compartilhadas com os demais docentes, criando assim, uma rede de padrões que auxilia os docentes no seu planejamento.

Por fim, Verpoorten, Poumay e Leclercq (2007), desenvolveram a ferramenta 8 Learning Events Model (8LEM), que serve para a elaboração de cursos on-line. Tem por objetivo diversificar as práticas pedagógicas, motivando alunos e professores a refletirem quanto aos métodos dos componentes curriculares já cursados. Os autores não apresentaram feedbacks dos usuários quanto ao processo metodológico.

A Figura 1 apresenta um resumo dos métodos e ferramentas mapeados. 
Figura 1 - Resultados: ferramentas e métodos

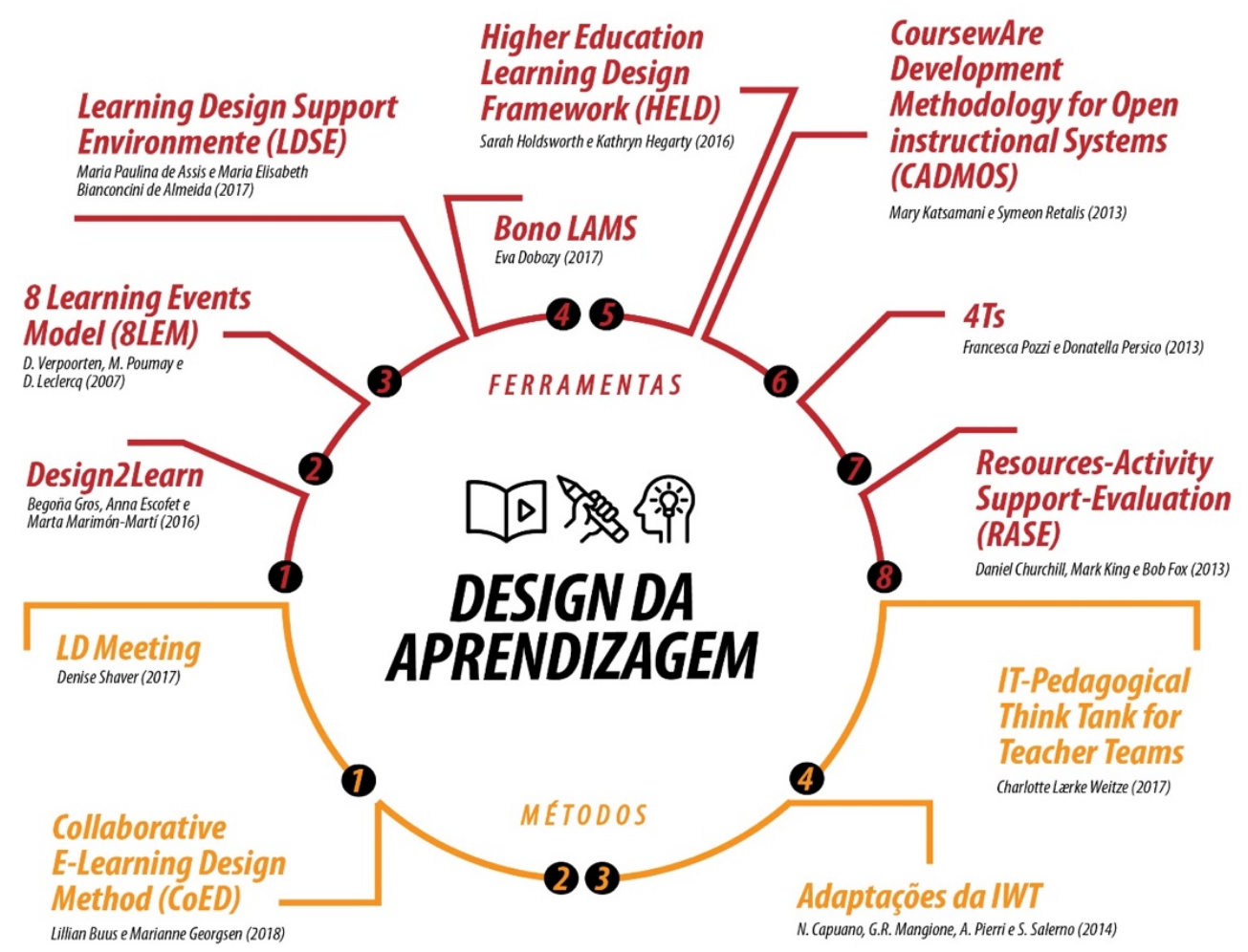

Fonte: Elaborado pela autora

A próxima sessão apresenta a discussão destes resultados.

\section{Discussão}

Tendo como ponto de partida o levantamento de ferramentas e métodos existentes para o processo de orientação na perspectiva dos estudos de Design da Aprendizagem, foram identificadas 12 possibilidades: oito ferramentas e quatro métodos.

Dentre as ferramentas e métodos encontrados, a maioria busca incentivar o protagonismo discente como intrínseco à prática e as atividades ou cursos elaborados, o que vai ao encontro da teoria do alinhamento construtivo de Biggs (1996). Característica esta, que está presente nas metodologias inovadoras, que tem o aluno como centro do processo de ensinoaprendizagem e vêm ganhando espaço no ensino superior. Também evidenciaram uma quebra de paradigma, uma vez que as propostas permitem a visualização do planejamento e isso favorece o repensar docente, promovendo a reflexão da docência diante das novas demandas da sociedade do século XXI.

O fazer docente, por meio da práxis, que prioriza a ação-reflexão-ação, deve ser repensado a todo momento. Novas competências são esperadas diante da dinamicidade da sociedade e assim o planejamento deve ser atualizado constantemente. Outro ponto relevante levantado com o uso das ferramentas e dos métodos é a possibilidade de visualizar as práticas, desde sua concepção, iniciando pelas competências previstas até a avaliação de forma sistemática, possibilitando a tomada de decisão mais assertivamente de acordo com o público-alvo que se pretende atingir. Os objetivos também ganham o papel primordial para a construção de atividades ou cursos, pois são pensados em uma escala crescente, buscando o aumento de complexidade ao longo do planejamento, de acordo com a Taxomomia de Bloom (BLOOM et. al. 1956).

Entretanto, foi possível verificar que dentre as ferramentas identificadas, o foco fica restrito, na maioria dos casos, ao desenvolvimento de atividades para inserção em 
ambientes virtuais de aprendizagem, muitas vezes adaptando os já existentes e utilizados em contexto presencial. Em contraponto, é muito citado nos relatos e feedbacks dos autores, a dificuldade dos docentes no uso de ferramentas on-line e na inclusão das tecnologias digitais em práticas educativas transcendo o uso apenas de computadores para pesquisas. Os resultados indicam a necessidade de possibilidades que instiguem a criatividade dos docentes e proporcionem o conhecimento de ferramentas, métodos e objetos de aprendizagem para serem utilizados. Esta característica é abordada apenas pela ferramenta Intelligent Web Teacher (IWT) (CAPUANO et. al., 2014).

Outro ponto relevante e que é uma das principais características do Design da Aprendizagem é a possibilidade de remixar as práticas existentes, premissa esta que não fica clara entre as ferramentas e métodos levantados. Apenas CoED (BUUS; GEORGSEN, 2018) e Design2lean (GROS; ESCOFET; MARTÍ, 2016) especificam esta possibilidade no momento do planejamento, ou seja, incentivam os docentes para que seus planos sejam compartilhados e que possam ser utilizados e ajustados sempre que necessários por outros docentes.

Em consonância, pouco se fala em compartilhamento dos designs elaborados, pois muitas vezes a orientação pode ser uma forma de representação para serem compartilhadas entre os pares. Apenas a ferramenta Learning Design Support Environment (LDSE) (ASSIS; ALMEIDA, 2017) indica esta possibilidade.

Também é possível verificar a falta de planejamentos coletivos entre os pares. Poucas ferramentas ou métodos oferecem esta possibilidade, mas também mostram a resistência dos professores na realização de atividades em conjunto. Desta forma, as ferramentas e os métodos não parecem auxiliar na promoção de propostas inter ou transdisciplinares. Apenas a ferramenta BONO Lams (DOBOZY, 2017) traz a transdisciplinaridade como diferencial ao planejamento. A ferramenta IT - Pedagogical Think Tank também visa o engajamento docente, porém sem mencionar a interdisciplinaridade, mas esta pode estar implícita ao processo.

\section{Considerações finais}

Este artigo teve como objetivo identificar quais as ferramentas e os métodos estão sendo utilizados no contexto do planejamento docente, a partir de estudos na área do Design da Aprendizagem.

Verificou-se que existem diferentes propostas para orientar o planejamento. Entretanto, ficou evidente a necessidade de incentivar, por meio da formação docente, o uso das tecnologias digitais em práticas educativas.

Os estudos apontaram que as ferramentas e os métodos propostos para apoiar o planejamento orientam o docente a observar o público-alvo (quem é o aluno?), bem como alinhar os objetivos para as atividades pretendidas, sendo coerentes com as competências que querem ser atingidas, resultando em um processo avaliativo adequado.

As atividades devem proporcionar o engajamento discente, fazendo com que os alunos se sintam implicados no seu processo formativo, como protagonista de sua aprendizagem. Os estudos também evidenciam a necessidade de promover $o$ planejamento em pares, incentivando a interdisciplinaridade.

Por fim, compreende-se a necessidade de promover a formação de professores no que diz respeito ao planejamento docente, por meio do Design da Aprendizagem. O planejamento apoiado em ferramentas e/ou métodos vêm ganhando espaço nas IES de forma global e se mostrando muito versátil, útil e disruptivo.

\section{Referências}


ASSIS, Maria Paulina de; ALMEIDA, Maria Elizabeth Bianconcini de. Learning Design e Tecnologias: Criação de Ambientes Colaborativos para a Aprendizagem. Psicologia da Educação, São Paulo, 44, $1^{\circ}$ sem. de 2017, pp. 47-56. Disponível em: $<$ http://pepsic.bvsalud.org/pdf/psie/n44/n44a05.pdf > . Acesso em: 18 maio 2020.

BASSANI, P.B.S. Documentação de atividades de aprendizagem com uso de tecnologias. In: $3^{\circ}$ Congresso Brasileiro de Informática na Educação (CBIE 2014) e Jornada de Atualização em Informática na Educação (JAIE), 2014. Anais. Disponível em: $<$ https://www.br-ie.org/pub/index.php/pie/article/view/3155> Acesso em: 11 dez. 2019

BIGGS, J. B. Enhancing teaching through constructive alignment. Higher Education, v.32, n 3, p. $347-364,1996$.

BLOOM, B. S. et al. Taxonomy of educational objectives. New York: David Mckay, 1956. 262 p. (v. 1)

BUUS, Lillian; GEORGSEN, Marianne. A Learning Design Methodology for Developing Short Learning Programmes in Further and Continuing Education. Journal of Interactive Media in Education; Vol 2018, No 1; 8 ; 1365-893X.

CAPUANO, Nicola; MANGIONE, Giuseppina Rita; PIERRI Anna; SALERNO, Saverio. Personalization and Contextualization of Learning Experiences based on Semantics. International Journal of Emerging Technologies in Learning (iJET), Vol 9, No 7 (2014). Disponível em:<https://online-journals.org/index.php/ijet/article/view/3666>. Acesso em: 18 maio 2020.

CHURCHILL, Daniel; KING Mark; FOX Bob. Learning design for science education in the 21 st century. Zbornik Instituta za pedagoska istrazivanja 2013 Volume 45, Issue 2, Pages: 404-421. Disponível em: <https://doi.org/10.2298/ZIPI1302404C> Acesso em: 18 maio 2020.

CONOLE, G. Designing for Learning in an Open World. Springer, New York, 2013.

DALZIEL, J., et al. A Declaração de Larnaca sobre Design de prendizagem. Journal of Interactive Media in Education, 2016. Disponível em: < http://doi.org/10.5334/ jime.407> Acesso em: 16 jul. 2020.

DOBOZY, E. The Pre-designed Lesson: Teaching with Transdisciplinary Pedagogical Templates (Tpts). Technology, Knowledge and Learning Volume 22, Issue 2, 1 July 2017, Pages 143-150.

GEORGSEN, Marianne. Engaging teaching professionals in design for on line learning. Springer-Verlag Berlin Heidelberg, 2011. Disponível em:

$<$ https://www.academia.edu/16225127/Engaging_teaching_professionals_in_design_for _on_line_learning?auto=download $>$ Acesso em: 18 maio 2020. 
, M; NYVANG. Collaborative e-Learning Design Method (CoED) (No.

12), e-Learning Lab Publication Series. Aalborg University, E-Learning Lab, 2007.

GROS Begoña; ESCOFET Anna; MARTÍ , Marta Marimón . Los patrones de diseño como herramientas para guiar la prática del professorado. RELATEC Revista Latinoamericana de Tecnología Educativa, Vol. 15(3) (2016) 1125. Disponível em: $<$ https://relatec.unex.es/article/view/2650/1923> Acesso em: 18 maio 2020.

HOLDSWORTH Sarah; HEGARTY, Kathryn. From praxis to delivery: a Higher Education Learning Design Framework (HELD) Journal of Cleaner Production 122 (2016) 176e185. Disponível em: $<$ https://www.sciencedirect.com/science/article/pii/S0959652616001232?via\%3Dih ub> Acesso em: 17 maio 2020.

KATSAMANI, Mary; RETALIS, Symeon. Orchestrating learning activities using the CADMOS learning design tool. A Research in Learning Technology (RLT), Vol 21 (2013). Disponível em: $<$ https://doi.org/10.3402/rlt.v21i0.18051> Acesso em: 18 maio 2020 .

KITCHENHAM, B.; CHARTERS, S.: Guidelines for performing systematic literature reviews in software engineering. Technical Report EBSE 2007-001, Keele University and Durham University Joint Report (2007)

MENEGOLLA, Maximiliano; SANT'ANNA, Ilza Martins. Por que planejar? Como planejar? Currículo, área, aula. Petrópolis, RJ: Vozes, 2014.

MORAN, José. Educação Híbrida um conceito chave para a educação, hoje. In BACICH, Lilian. Ensino híbrido personalização e tecnologia na educação. Porto Alegre: Bookman 2015

POZZI, Francesca; PERSICO, Donatella. Sustaining learning design and pedagogical planning in CSCL. A Research in Learning Technology (RLT), Vol 21 (2013). Disponível em: $<$ https://doi.org/10.3402/rlt.v21i0.17585> Acesso em: 18 maio 2020

SHAVER, Denise. The Added Value of Conducting Learning Design Meeting to the Online Course Development Process. TechTrends: Linking Research \& Practice to Improve Learning is a copyright of Springer, 2017. All Rights Reserved. Disponível em: <https://eds.a.ebscohost.com/eds/pdfviewer/pdfviewer?vid=1\&sid=bb17a69f-6a684141-9ccf-680872469d49\%40sdc-v-sessmgr01> Acesso em: 17 maio 2020.

THE LARNACA DECLARATION ON LEARNING DESIGN. Disponível em: https://larnacadeclaration.wordpress.com/. 2013.

VERPOORTEN D.; POUMAY M.; LECLERCQ, D. The 8 Learning Events Model: a Pedagogic Conceptual Tool Supporting Diversification of Learning Methods.

Interactive Learning Environments, Aug. 2007, Vol 15, Issue 2, pages $151-160$. Disponível em: <https://orbi.uliege.be//handle/2268/10129\#?>. Acesso em: 18 maio 2020.

WEITZE, Charlotte Lærke. Designing pedagogical innovation for collaborating teacher teams. Journal of Education for Teaching, 2017. Disponível em: $<10.1080 / 02607476.2017 .1319511>$ Acesso em: 18 maio 2020. V. $18 \mathrm{~N}^{\circ} 1$, julho, 2020 RENOTE DOI: 